\title{
Evidence of a pure starburst nature of the nuclear region of NGC 253
}

\author{
A. Brunthaler ${ }^{1}$, P. Castangia ${ }^{1,2}$, A. Tarchi ${ }^{2,3}$, C. Henkel $^{1}$ M. J. Reid ${ }^{4}$, H. Falcke ${ }^{5,6}$, and K. M. Menten ${ }^{1}$ \\ 1 Max-Planck-Institut für Radioastronomie, Auf dem Hügel 69, 53121 Bonn, Germany \\ e-mail: brunthal@mpifr-bonn.mpg.de \\ 2 INAF - Osservatorio Astronomico di Cagliari, Loc. Poggio dei Pini, Strada 54, 09012 Capoterra (CA), Italy \\ INAF - Istituto di Radioastronomia, via Gobetti 101, 40129 Bologna, Italy \\ 4 Harvard-Smithsonian Center for Astrophysics, 60 Garden Street, Cambridge, MA 02138, USA \\ 5 Department of Astrophysics, Radboud Universiteit Nijmegen, Postbus 9010, 6500 GL Nijmegen, The Netherlands \\ 6 ASTRON, Postbus 2, 7990 AA Dwingeloo, The Netherlands
}

Received 2 January 2009 / Accepted 30 January 2009

\section{ABSTRACT}

\begin{abstract}
We present high-resolution spectral line and continuum VLBI and VLA observations of the nuclear region of NGC 253 at $22 \mathrm{GHz}$. While the water vapor masers in this region were detected on arcsecond and milliarcsecond scales, we could not detect any compact continuum emission with a $5 \sigma$ upper limit of $\sim 1 \mathrm{mJy}$. The observations reveal that the water maser emission is not related to a possible low-luminosity active galactic nucleus but is almost certainly associated with star-formation activity. Not detecting any compact continuum source on milliarcsecond scales also questions the presence of a - previously assumed - active nucleus in NGC 253.
\end{abstract}

Key words. techniques: interferometric - masers - galaxies: starburst - galaxies: individual: NGC 253

\section{Introduction}

Extragalactic water masers in the $22.23508 \mathrm{GHz}$ transition between the $6_{16}$ and $5_{23}$ rotational levels of ortho- $\mathrm{H}_{2} \mathrm{O}$ are found in star-forming regions (e.g., M33: Churchwell et al. 1977 1977, LMC: Scalise \& Braz 1981, IC 10: Becker et al. 1993; Castangia et al. 2008) and associated with an active galactic nucleus (AGN), either in accretion disks (e.g., NGC 4258, Miyoshi et al. 1995; UGC 3789, Reid et al. 2008), cloud-jet interactions (e.g., NGC 1052, Claussen et al. 1998; NGC 1068, Gallimore 2001; Mrk 348, Peck et al. 2003), or nuclear outflows (Circinus, Greenhill et al. 2003). While the star forming masers are only detected in relatively nearby galaxies, the powerful AGN masers can be observed out to cosmological distances (Barvainis \& Antonucci 2005; Impellizzeri et al. 2008). Extragalactic water masers are a powerful tool for investigating the nuclear environment of active galaxies and have also been used to measure the proper motions of members of the local group of galaxies (Brunthaler et al. 2005b; Brunthaler et al. 2007).

One of the nearest water masers outside the local group that can be used for proper motion studies is located in NGC 253, a prominent spiral galaxy in the nearby Sculptor group of galaxies. It has been known for more than 20 years (Ho et al. 1987) and is associated with the galaxy's mildly starbursting nuclear environment (Henkel et al. 2004). NGC 253 is commonly placed at a distance of $2.5 \mathrm{Mpc}$, but some studies favor smaller ( $\leq 2.3 \mathrm{Mpc}$ : Davidge \& Pritchet 1990) or larger (3.5 Mpc: Rekola et al. 2005; 3.3 Mpc: Mouhcine et al. 2005) distances. Throughout the paper we adopt the recent tip of the red giant branch distance estimate of $3.3 \mathrm{Mpc}\left(1^{\prime \prime}=16 \mathrm{pc}\right.$ or $\left.1 \mathrm{mas}=3300 \mathrm{AU}\right)$. At this distance, one could measure the proper motion of NGC 253 with an accuracy of $\sim 50 \mathrm{~km} \mathrm{~s}^{-1}$ within a few years.
Radio continuum images of NGC 253 with arcsecond resolution show several compact radio sources (Ulvestad \& Antonucci 1997, hereafter UA97). The strongest source (TH2 in UA97) near the dynamical center of the galaxy is unresolved and may represent a low-luminosity active galactic nucleus (LLAGN) which is not dominating the nuclear environment. VLA A configuration observations at $22 \mathrm{GHz}$ (Hofner et al. 2006, hereafter HBT06) have shown that the maser is not associated with $\mathrm{TH} 2$ but with another nearby continuum source (TH4 in UA97), which is probably a supernova remnant.

Here we present deep $22 \mathrm{GHz}$ VLBI and VLA observations with milliarcsecond (mas) and arcsecond resolution of the water maser and the continuum sources in the central region of NGC 253.

\section{Observations and data reduction}

\subsection{VLBI observations}

We observed NGC 253 with the NRAO $^{1}$ Very Long Baseline Array (VLBA) and the phased Very Large Array (VLA) in its C configuration under program BB254 on 2008 March 24. We observed in two $16 \mathrm{MHz}$ wide bands in dual circular polarization each. The 256 spectral channels in each band yielded a channel spacing of $62.5 \mathrm{kHz}$, equivalent to $0.84 \mathrm{~km} \mathrm{~s}^{-1}$, and covered a velocity range of $216 \mathrm{~km} \mathrm{~s}^{-1}$. One frequency band was centered at an LSR velocity of $120 \mathrm{~km} \mathrm{~s}^{-1}$ (the peak of the maser emission), while the second band was centered at an LSR velocity of $-95.72 \mathrm{~km} \mathrm{~s}^{-1}$.

\footnotetext{
1 The National Radio Astronomy Observatory is a facility of the National Science Foundation operated under cooperative agreement by Associated Universities, Inc.
} 
The observations involved rapid switching for $6 \mathrm{~h}$ between the phase-calibrator VCS1 J0038-2459 from the VLBA Calibrator Survey (Beasley et al. 2002), which is a compact background continuum source, and the target source NGC 253. We switched sources every $50 \mathrm{~s}$ and achieved on-source integration times of $\sim 42 \mathrm{~s}$ for the VLBA antennas and $\sim 20 \mathrm{~s}$ for the slower VLA antennas. 3C 454.3 was also observed as fringe finder.

Before and after the phase referencing observation we included geodetic-like observations where we observed for $60 \mathrm{mn}$ $\sim 15$ strong radio sources $(>200 \mathrm{mJy}$ ) with accurate positions $(<1$ mas) at different elevations to estimate an atmospheric zenith delay error in the VLBA calibrator model (see Reid \& Brunthaler 2004 and Brunthaler et al. 2005b, for a discussion). Since the atmospheric zenith delay should be very similar for all VLA antennas, we used only a single VLA antenna in the geodetic-like observations. The other antennas were used to observe NGC 253 in spectral line mode (see the end of Sect. 2.2).

The VLBI data were edited and calibrated using standard programs in the astronomical image processing system (AIPS). First, we applied the latest values of the Earth's orientation parameters. Zenith delay corrections were performed based on the results of the geodetic-like observations. A-priori amplitude calibration was applied using system temperature measurements and standard gain curves. We performed a "manual phasecalibration" using the data from 3C 454.3 to remove instrumental phase offsets among the two frequency bands. Then, we fringe fitted the data from VCS1 J0038-2459. Since the a priori amplitude calibration of the phased VLA was off by a large factor, we performed two steps of amplitude self-calibration on VCS1 J0038-2459. First, we changed only the gain of the VLA and included all antennas in the second step. Finally, we transferred the calibration to our target source NGC 253.

\subsection{VLA observations}

NGC 253 was observed with the VLA in its most extended A configuration under program AC753 on 2004 November 2. We employed a single band of $12.5 \mathrm{MHz}$ subdivided in 64 channels of width $195.3 \mathrm{kHz}$, yielding a channel spacing of $2.63 \mathrm{~km} \mathrm{~s}^{-1}$ and covered a velocity range of $168 \mathrm{~km} \mathrm{~s}^{-1}$. The observing band was centered at the frequency corresponding to the LSR velocity $\left(120 \mathrm{~km} \mathrm{~s}^{-1}\right)$ of the main maser component. The absolute amplitude calibration was obtained using $3 \mathrm{C} 48\left(S_{22 \mathrm{GHz}}=1.13 \mathrm{Jy}\right)$, while as phase calibrator we employed the compact source 0120-270 $\left(S_{22 \mathrm{GHz}}=0.54 \mathrm{Jy}\right)$. The total observing time was $4.5 \mathrm{~h}$. We conducted pointing observations regularly and employed fast switching with $170 \mathrm{~s}$ on the target source and $70 \mathrm{~s}$ on the phase calibrator.

The data were calibrated in the standard manner using AIPS. The continuum emission was subtracted from the spectral line data by fitting a straight line to the visibilities of the line free channels with the task UVLSF. This task also uses the fitted baseline to construct a line-free uv-dataset that was used to create a continuum map. Both line and continuum datasets were Fourier-transformed using natural weighting and then deconvolved utilizing the CLEAN algorithm.

The data from the phased VLA in our VLBI observation on 2008 March 24, obtained with the VLA in C configuration, were also correlated in normal VLA continuum mode with two frequency bands of $50 \mathrm{MHz}$ width in dual polarization. The data were calibrated using standard techniques in AIPS. 3C 48 was used for the flux density calibration. VCS1 J0038-2459 was then used as amplitude and phase calibrator before we performed two rounds of phase self-calibration and one phase \& amplitude self-calibration on NGC 253.

Since we used only a single VLA antenna during the geodetic-like observations, we could simultaneously observe NGC 253 with the remaining antennas in spectral line mode. We used a total bandwidth of $12.5 \mathrm{MHz}$ and 64 spectral channels, yielding a channel spacing of $2.63 \mathrm{~km} \mathrm{~s}^{-1}$ and covered a velocity range of $168 \mathrm{~km} \mathrm{~s}^{-1}$. We employed fast switching between NGC 253 and the nearby phase calibrator 0120-270. The total integration time on NGC 253 was 24 minutes. The data were also calibrated using standard techniques in AIPS. 3C 48 was used for the flux density calibration. After amplitude and phase calibration on $0120-270$ we performed first a phase self-calibration and then a phase \& amplitude self-calibration using our image from the continuum observation. Finally, we subtracted the continuum emission from the uv-data set.

\section{Results}

\subsection{VLBI Data}

The VLBI data were imaged with a Gaussian uv-taper of $350000 \mathrm{k} \lambda$ and natural weighting to obtain the highest brightness sensitivity. We mapped two fields of $0.4 \times 0.4$ arcsecond $^{2}$ centered at the positions of the VLA components TH2 and TH4. First, all channels in the line free frequency band were averaged to image the continuum emission of NGC 253. The restoring beam was $2.44 \times 1.11 \mathrm{mas}^{2}$ with a position angle of $12^{\circ}$. No continuum emission was detected with a $5 \sigma$ upper limit of $1.1 \mathrm{mJy}$ for both fields. Since no maser is known in the field of $\mathrm{TH} 2$, we imaged this field also with both frequency bands to obtain a deeper image. The restoring beam was $2.63 \times 1.31$ mas $^{2}$ with a position angle of $14^{\circ}$. No continuum emission was detected with a $5 \sigma$ upper limit of $0.8 \mathrm{mJy}$ from $\mathrm{TH} 2$.

The frequency band with the maser emission was imaged channel by channel in a field of $0.2 \times 0.2 \operatorname{arcsecond}^{2}$ centered at the position of TH4. The restoring beam was $1.98 \times 0.95$ mas $^{2}$ with a position angle of $2.3^{\circ}$. We reached a noise level of $\sim 2.5 \mathrm{mJy}$ in a single spectral channel. We detected maser emission from two spatially distinct regions separated by 7.4 mas or 0.12 pc (VLBI-East and VLBI-West). A velocity integrated maser map is shown in Fig. 1.

The spectrum of the western feature (Fig. 1, upper panel) shows two narrow components $\left(2-5 \mathrm{~km} \mathrm{~s}^{-1}\right)$ superimposed on a broad $\sim 45 \mathrm{~km} \mathrm{~s}^{-1}$ wide component. The spectrum of the eastern feature (Fig. 1, lower panel) shows three narrow $\left(2-4 \mathrm{~km} \mathrm{~s}^{-1}\right)$ components, one with a flux density of $\sim 30 \mathrm{mJy}$ and two with flux densities of $\sim 10 \mathrm{mJy}$. The parameters of Gaussian fits to the lines are summarized in Table 1. Please note that the width of the line at $v_{\mathrm{LSR}}=114 \mathrm{~km} \mathrm{~s}^{-1}$ in the eastern component was fixed in the fit, since otherwise the component becomes very broad (33 $\mathrm{km} \mathrm{s}^{-1}$, probably caused by the slightly higher noise in the spectrum of the eastern component at the velocity of the peak of the western component at $117 \mathrm{~km} \mathrm{~s}^{-1}$ ). The positions of the emission from the three components in each spectrum are consistent within 0.2 mas, the error being dominated by the position uncertainties of the weaker features.

The estimated absolute position of the strongest maser in the western feature is $00^{\mathrm{h}} 47^{\mathrm{m}} 33.16974^{\mathrm{s}}-25^{\circ} 17^{\prime} 17.4734^{\prime \prime}(\mathrm{J} 2000)$ with an uncertainty (the error is dominated by the uncertainty in the position reference) of 0.23 and 0.49 mas in right ascension and declination, respectively. This is consistent within 30 mas (or $\sim 0.5 \mathrm{pc}$ ) with the position of the continuum source TH4 from the VLA observations (see Sect. 3.2.1). This confirms the 


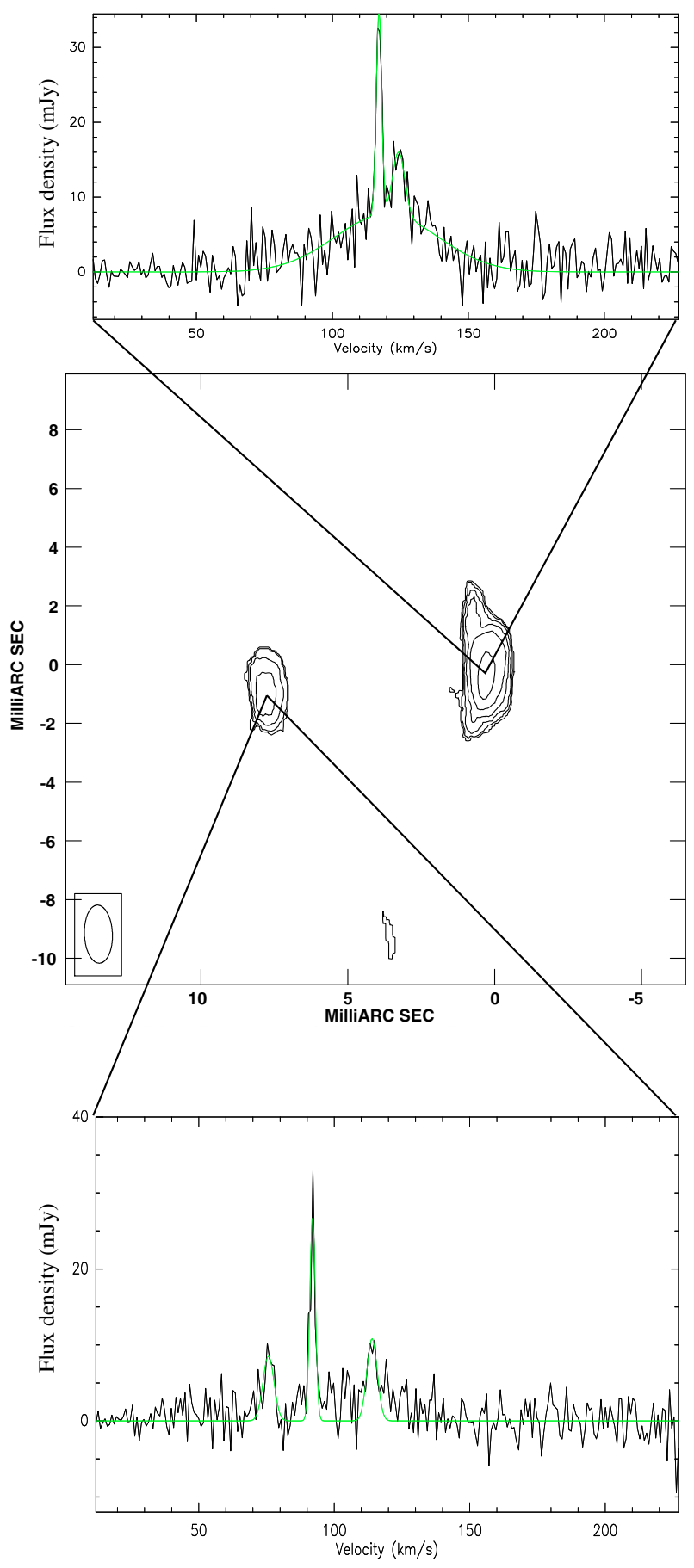

Fig. 1. Middle panel: velocity integrated water maser emission in NGC 253 from the VLBI observation on 2008 March 24 at the position of TH4. The contours start at $7 \mathrm{mJy} \mathrm{km} \mathrm{s}^{-1}$ and increase with factors of 2. Upper panel: spectrum of the western feature, containing one broad and two narrow components. Lower panel: spectrum of the eastern feature, containing three narrow components.

result of HBT06 that the maser is associated with the continuum source TH4.

\subsection{VLA Data}

\subsubsection{November 2 (A configuration)}

We detected water maser emission in NGC 253 at $V_{\mathrm{LSR}} \sim$ $120 \mathrm{~km} \mathrm{~s}^{-1}$ with a peak flux density of $\sim 70 \mathrm{mJy}$ (Fig. 2, lower panel). The maser line is confidently identified with the $\mathrm{H}_{2} \mathrm{O}$ component reported by Henkel et al. (2004). The line parameters, obtained from a Gaussian fit, are reported in Table 1. The line integrated intensity of $\sim 2.1 \mathrm{Jy} \mathrm{km} \mathrm{s}^{-1}$ yields an $\mathrm{H}_{2} \mathrm{O}$ isotropic luminosity of $\sim 0.53 L_{\odot}$.

We produced a continuum map using the line free channels (Fig. 2). The synthesized beam was $0.18 \times 0.09 \operatorname{arcsec}^{2}$ with a position angle of $3^{\circ}$. We detected 9 compact radio sources most of which can be identified with the brightest compact components reported in the nuclear region of NGC 253 by UA97. One further source is present between TH8 and TH9 that was also detected by UA97 but had never assigned a label likely because of its weakness. Among the bright UA97 sources, only TH5 is not seen in our map. We fitted elliptical Gaussians to the source brightness distributions and derived the peak brightnesses and integrated flux densities as well as the angular dimensions. The results of these fits are reported in Table 2.

\subsubsection{March 24 (C configuration)}

From the continuum data, we imaged a field of $102 \times 102 \mathrm{arcsec}^{2}$, covering almost the full primary beam of a single VLA antenna. The restoring beam was $1.60 \times 0.85 \operatorname{arcsec}^{2}$ with a position angle of $-5^{\circ}$. The $22 \mathrm{GHz}$ continuum emission of NGC 253 is shown in Fig. 2. We achieved an image rms of $0.11 \mathrm{mJy}^{\text {beam }}{ }^{-1}$ which is the deepest continuum image of NGC 253 at $22 \mathrm{GHz}$ so far. We also detected 3 compact sources that were not seen in the higher resolution maps of UA97 at 22 GHz: 4.81-43.60, 6.4037.1, and 6.81-29.6 (using nomenclature of UA97, see Table 2).

From the line data, we imaged also a field of $102 \times$ $102 \operatorname{arcsec}^{2}$ and achieved an image rms of $12 \mathrm{mJy}$. The restoring beam was $1.52 \times 1.30 \operatorname{arcsec}^{2}$ with a position angle of $-11.61^{\circ}$. No maser emission was detected at a $5 \sigma$ upper limit of $60 \mathrm{mJy}$, indicating a lower flux level of the maser compared to previous observations.

\section{Discussion}

\subsection{Continuum emission}

The assumed nucleus of NGC 253 (TH2) has a brightness temperature greater than $20000 \mathrm{~K}$ at $22 \mathrm{GHz}$ and greater than $40000 \mathrm{~K}$ at $15 \mathrm{GHz}$ (UA97). Chandra observations of the nuclear region show evidence for an intermediate mass black hole or a low-luminosity AGN (LLAGN) component, but the spatial resolution is not sufficient to separate TH4 and TH2 (Weaver et al. 2002). The X-ray emission is also consistent with emission from ultra-luminous X-ray sources in other galaxies. Hence, it is still not clear whether the radio source $\mathrm{TH} 2$ is indeed an LLAGN. Recently, Fernández-Ontiveros et al. (2008) questioned the presence of an AGN component because of the lack of any optical or IR counterpart.

Variability of TH2 or TH4 would point toward an AGN nature of these radio sources. A comparison of our measured flux densities with values in the literature is difficult due to potential contamination by the extended diffuse emission from the galaxy. Different resolutions will lead to different flux density estimates for the same source. This can be seen in the comparison of the flux densities from A and B configuration data in UA97. The beam size in our observation is $\sim 40 \%$ larger than the beam size in the A configuration observation of UA97 which explains why we find larger fluxes for all sources. Our flux density of $23.6 \mathrm{mJy}$ for $\mathrm{TH} 2$ is consistent with the $21.5 \mathrm{mJy}$ found in UA97, indicating no strong variability. However, HBT06 report a flux density 
Table 1. Details of the detected water maser components in our VLBI and VLA A-Array observations.

\begin{tabular}{lccccc}
\hline \hline Source & $\begin{array}{c}v_{\mathrm{LSR}} \\
{\left[\mathrm{km} \mathrm{s}^{-1}\right]}\end{array}$ & $\begin{array}{c}\text { Width } \\
{\left[\mathrm{km} \mathrm{s}^{-1}\right]}\end{array}$ & $\begin{array}{c}\text { Peak flux } \\
{[\mathrm{mJy}]}\end{array}$ & $\begin{array}{c}\text { Integrated flux } \\
{\left[\mathrm{Jy} \mathrm{km} \mathrm{s}^{-1}\right]}\end{array}$ & $\begin{array}{c}L_{\mathrm{H}_{2} \mathrm{O}} \\
{\left[L_{\odot}\right]}\end{array}$ \\
\hline VLBI-West & $117.1 \pm 0.1$ & $2.3 \pm 0.2$ & 27.4 & $0.068 \pm 0.007$ & 0.017 \\
& $119.9 \pm 1.5$ & $44.6 \pm 6.3$ & 7.7 & $0.366 \pm 0.026$ & 0.092 \\
& $124.4 \pm 0.4$ & $5.3 \pm 1.3$ & 8.5 & $0.048 \pm 0.014$ & 0.012 \\
VLBI-East & $75.7 \pm 0.6$ & $4.8 \pm 1.5$ & 8.5 & $0.043 \pm 0.009$ & 0.011 \\
& $92.0 \pm 0.1$ & $2.4 \pm 0.3$ & 27.2 & $0.070 \pm 0.006$ & 0.017 \\
& $114.0 \pm 0.4$ & 4.5 & 10.8 & $0.052 \pm 0.006$ & 0.013 \\
VLBI total & & & & $0.647 \pm 0.033$ & 0.162 \\
VLA & $116.8 \pm 0.5$ & $35 \pm 1$ & & $2.10 \pm 0.06$ & 0.53 \\
\hline
\end{tabular}

of only $\sim 8 \mathrm{mJy}$ for TH2 (VLA A configuration, and a synthesized beam comparable to our A configuration beam). On the other hand, our flux density for TH4 of $12.5 \mathrm{mJy}$ is comparable to the values found by HBT06 (8 $\mathrm{mJy}$ ), but larger than the value reported in UA97 (3.78 mJy in A configuration and $9.69 \mathrm{mJy}$ in $\mathrm{B}$ configuration). Thus, taking into account the different resolutions in the observations, there is no clear evidence for variability of TH4 but signs of possible variability of TH2. Multi-epoch observations with the VLA with identical resolutions are needed to decide this question.

The continuum source $\mathrm{TH} 2$, the presumed nucleus, is unresolved with the VLA with a source size $<100$ mas and a flux density of $\sim 20 \mathrm{mJy}$, but completely resolved by our VLBI observation. Tingay (2004) did not detect TH2 with the Australian Long Baseline Array (LBA) at $1.4 \mathrm{GHz}$ probably due to strong free-free absorption. More recently, Lenc \& Tingay (2006) performed higher frequency LBA observations at $2.3 \mathrm{GHz}$, detecting six sources. TH2 was detected with a flux density of $\sim 6 \mathrm{mJy}$, a source size of $80 \times 60$ mas, and a brightness temperature of $\sim 10^{5} \mathrm{~K}$. This value is not particularly unusual and can be justified as well in terms of radio supernova or a young supernova remnant without necessarily invoking the presence of AGN activity. The spectrum of the source can be fit with a free-free absorbed-power-law.

One possible reason for our non detection of $\mathrm{TH} 2$ could be scatter broadening similar to Sgr A* (e.g. Krichbaum et al. 1993; Yusef-Zadeh et al. 1994). Assuming a $\lambda^{2}$ law and a source size of $80 \times 60$ mas at $2.3 \mathrm{GHz}$, Lenc \& Tingay (2006), one would expect a source size of $\sim 0.86 \times 0.64$ mas at $22 \mathrm{GHz}$, which is smaller than our beam size. Hence, we can rule out scatter broadening.

Since TH2 has a turnover frequency between 4.8 and $8 \mathrm{GHz}$ (UA97), it could be a GHz peaked spectrum (GPS) source, i.e. a very young AGN. However, the linear size vs. turnover frequency relation (O'Dea \& Baum 1997 predicts a size of $\sim 20 \mathrm{pc}$, more than 12 times larger than the upper limit of $1.6 \mathrm{pc}$ from the VLA observations. Nevertheless, there is considerable scatter in the correlation and at least one GPS source is known with a turnover frequency of $10 \mathrm{GHz}$ and a projected size of $<1 \mathrm{pc}$ (Brunthaler et al. 2000; Brunthaler 2005a). Hence, we cannot rule out a GPS scenario for NGC 253, although GPS sources are usually several orders of magnitudes more powerful than TH2. The existence of an inactive supermassive black hole like Sgr A* cannot be ruled out by our observation, since its flux density at a distance of $3.3 \mathrm{Mpc}$ would be only $6 \mu \mathrm{Jy}$.

Lenc \& Tingay 2006 did not detect the source TH4, the continuum source associated with the water maser emission, at $2.3 \mathrm{GHz}$ with the LBA, excluding with high confidence that TH4 is an AGN. UA97 suggest that TH4 is a SNR mostly because its radio spectral index $\left(\alpha_{1.3 \mathrm{~cm}}^{3.6 \mathrm{~cm}}=-0.52 \pm 0.17\right)$ is typical for optically thin synchrotron emission.

\section{2. $\mathrm{H}_{2} \mathrm{O}$ maser emission}

The upper limit of $60 \mathrm{mJy}$ for maser emission in our VLA data that was taken during our VLBI observation in March 2008 shows that the maser (which we detected at about $30 \mathrm{mJy}$ ) was observed during a weak period. The spectrum of the eastern component in our VLBI observation resembles remarkably well the spectrum of the Galactic water maser in IC 1396N (Slysh et al. 1999) where the blue-shifted and redshifted lines are found 410 and $10^{4}$ AU from the position of the protostar and are interpreted as an outflow. If one would interpret the spectrum as a rotating disk, a rotation velocity of $\sim 19 \mathrm{~km} \mathrm{~s}^{-1}\left(\frac{114-76}{2} \mathrm{~km} \mathrm{~s}^{-1}\right)$ at a radius of $\leq 330 \mathrm{AU}$ yields an enclosed mass of $\leq 270 \sin ^{-2}$ i $M_{\odot}$. Although the current data is not sufficient to distinguish between these two scenarios, the emission is comparable with water masers in Galactic star forming regions. This is in contrast to the broad emission $\left(\sim 45 \mathrm{~km} \mathrm{~s}^{-1}\right)$ in the western maser component, which is not typical for water masers in star forming regions. However, this broad component could be also the sum of several narrow components which blend together.

A comparison between the VLA A-array maser feature, observed by us in November 2004, with the one detected by Henkel et al. (2004) using the CnB configuration in September 2002 shows that both the line profile and the center velocity of the maser features are consistent. However, the integrated flux density and, as a consequence, the total isotropic luminosity of the line in 2004 are weaker by a factor of two than in 2002. Nevertheless, the line in our observation (see Table 1) is stronger than the line seen in 1997 (1.65 Jy km s${ }^{-1}$, VLA CnB configuration) and 1998 (0.54 Jy km s${ }^{-1}$,VLA A configuration) by HBT06. Thus, the maser exhibits significant variability on timescales of years.

\subsection{Association of the maser emission}

The water maser lines are blue shifted on the order of 100$200 \mathrm{~km} \mathrm{~s}^{-1}$ with respect to the velocity of the gas in the masing region. This has been interpreted according to different scenarios that mostly depend on the presence or absence of AGN activity in the nuclear region of NGC 253. The discrepancy in velocity might be explained by the presence of a circumnuclear accretion disk or an AGN-related nuclear outflow. However, as described in Sect. 4.1, it is questionable whether NGC 253 hosts an AGN. Since the water maser is clearly associated with TH4 (HBT06; present work), this suggests that a maser-AGN relation is highly unlikely and that the maser emission is almost surely related to star formation activity. 
Table 2. Details of the detected compact VLA sources in our VLA observations on 2004 November 2 (A configuration) and 2008 March 24 (C configuration). Shown are also the flux densities at $22 \mathrm{GHz}$ from UA97 from their observations with the A and B configuration.

\begin{tabular}{|c|c|c|c|c|c|c|c|c|c|c|}
\hline Config & Source & $\begin{array}{c}\alpha_{J 2000} \\
00^{\mathrm{h}} 47^{\mathrm{m}}\end{array}$ & $\begin{array}{c}\delta_{J 2000} \\
-25^{\circ} 17^{\prime} \\
\end{array}$ & $\begin{array}{c}\text { Major axis } \\
{\left[{ }^{\prime \prime}\right]}\end{array}$ & $\begin{array}{c}\text { Minor axis } \\
{\left[{ }^{\prime \prime}\right]}\end{array}$ & $\begin{array}{l}\text { P.A. } \\
{\left[{ }^{\circ}\right]}\end{array}$ & $\begin{array}{c}\text { Peak flux } \\
\text { [mJy] }\end{array}$ & $\begin{array}{c}\text { Integrated flux } \\
{[\mathrm{mJy}]}\end{array}$ & $\begin{array}{c}S_{v}(\mathrm{VLA}-\mathrm{A}) \\
{[\mathrm{mJy}]}\end{array}$ & $\begin{array}{c}S_{v}(\mathrm{VLA}-\mathrm{B}) \\
{[\mathrm{mJy}]}\end{array}$ \\
\hline $\bar{A}$ & TH1 & $33^{\mathrm{s} .30}$ & $15^{\prime \prime} .54$ & $0.24 \pm 0.02$ & $0.17 \pm 0.02$ & $16 \pm 10$ & $3.8 \pm 0.4$ & $9.1 \pm 1.2$ & 2.09 & 6.72 \\
\hline A & TH2 & 33.18 & $17 " .15$ & $0.22 \pm 0.004$ & $0.13 \pm 0.002$ & $4 \pm 1$ & $23.6 \pm 0.4$ & $38.4 \pm 0.9$ & 21.53 & 35.79 \\
\hline A & TH4 & 33.17 & $17 " .48$ & $0.26 \pm 0.007$ & $0.16 \pm 0.005$ & $6 \pm 2$ & $12.5 \pm 0.4$ & $29.9 \pm 1.2$ & 3.78 & 9.69 \\
\hline A & TH6 & 33.11 & $188^{\prime \prime} 16$ & $0.40 \pm 0.04$ & $0.24 \pm 0.02$ & $176 \pm 7$ & $3.9 \pm 0.4$ & $21.9 \pm 2.3$ & 0.94 & 7.88 \\
\hline A & TH7 & $33^{s} .01$ & 19.42 & $0.26 \pm 0.03$ & $0.19 \pm 0.02$ & $15 \pm 13$ & $3.7 \pm 0.4$ & $10.7 \pm 1.4$ & 0.88 & 5.84 \\
\hline A & TH8 & $32^{s} .98$ & $19 ! .73$ & $0.28 \pm 0.03$ & $0.20 \pm 0.02$ & $21 \pm 11$ & $3.9 \pm 0.4$ & $13.0 \pm 1.5$ & 1.82 & 5.72 \\
\hline A & & 32.93 & $20 ! 31$ & $0.26 \pm 0.04$ & $0.25 \pm 0.04$ & $48 \pm 90$ & $2.4 \pm 0.4$ & $9.2 \pm 1.7$ & - & - \\
\hline A & TH9 & $32^{\mathrm{s}} .88$ & 21 ". 38 & $0.21 \pm 0.01$ & $0.13 \pm 0.006$ & $177 \pm 4$ & $7.8 \pm 0.4$ & $13.1 \pm 0.9$ & 3.57 & 9.79 \\
\hline $\mathrm{C}$ & $4.81-43.6$ & $32^{\mathrm{s}} .22$ & $21^{\prime \prime} .7$ & $3.5 \pm 0.7$ & $1.1 \pm 0.2$ & $1 \pm 5$ & $0.5 \pm 0.1$ & $1.4 \pm 0.4$ & - & - \\
\hline $\mathrm{C}$ & $6.40-37.1$ & 33.79 & $155^{\prime \prime} 3$ & $1.5 \pm 0.2$ & $0.9 \pm 0.1$ & $177 \pm 9$ & $0.9 \pm 0.1$ & $0.8 \pm 0.2$ & - & - \\
\hline $\mathrm{C}$ & $6.81-29.6$ & 34.20 & $08 . .1$ & $1.9 \pm 0.3$ & $0.8 \pm 0.1$ & $8 \pm 6$ & $0.8 \pm 0.1$ & $0.9 \pm 0.2$ & - & - \\
\hline
\end{tabular}

\subsubsection{Association with the supernova remnant TH4?}

Given the spatial coincidence of the maser emission with TH4 $(0.5 \mathrm{pc})$, which shows characteristics compatible with those of an SNR (Sect. 4.1), an association of the masing gas with the expanding shell of a SNR may seem promising. The anomalous velocity of the maser line would be due to the motion of the approaching side of the shell. However, the possibility that the maser is excited by the SNR itself is unlikely, since Galactic water masers have never been detected towards SNRs. A search for water masers in the three SNRs, W28, W44, and IC 443, yielded no detections (Claussen et al. 1999). The authors explained the non detections as a consequence of low post-shock densities found in the shocked regions of the SNRs, which are too low to produce a strong enough inversion of the $22 \mathrm{GHz}$ maser line. However, the SNR could play an indirect role in the exciting mechanism. The young stellar object responsible for the excitation of the maser might be born in a star forming region induced by the supernova shock wave like the scenario proposed for the Galactic cometary HII region G34.3+0.2 (Reid \& Ho 1985) which also host water masers (e.g. Fey et al. 1994) and is believed to interact with a wind from the precursor of the supernova remnant W44. Alternatively, the masing gas can be associated only in projection with the SNR. Here the line can result from amplification of the radio continuum background from the SNR as suggested by HBT06. However, in this case the masing gas should be still very close to the SNR shell to experience the "kick" or the line velocity issue would still remain open.

\subsubsection{Association with peculiar gas components?}

Other signs of peculiar kinematic gas components in NGC 253 have been reported in a number of recent papers. A bar-like structure, covering the inner $150^{\prime \prime}(2.4 \mathrm{kpc})$ region of NGC 253 was first observed in the near-infrared (Scoville et al. 1985; Forbes \& Depoy 1992) and confirmed by subsequent optical and mid-infrared observations (Forbes \& Depoy 1992; Pina et al. 1992). Counterparts of this stellar bar have been also found in CO (Canzian et al. 1988), HCN (Paglione et al. 1995), and CS (Peng et al. 1996). Observations in the radio recombination lines (RRL) H92 $\alpha$ and H53 $\alpha$ at higher spatial resolution revealed a velocity field in the central $10^{\prime \prime}(160 \mathrm{pc})$ that is discrepant with that derived from the lower resolution $\mathrm{CO}, \mathrm{CS}$, and $\mathrm{HCN}$ observations (Anantharamaiah \& Goss 1996; Rodriguez-Rico et al. 2006).

To explain the different kinematics observed for the ionized (traced by the RRLs) and the molecular gas (CO, HCN, and
CS), three alternative scenarios have been proposed including the presence of a secondary bar within the primary one, a compact supermassive object in the nuclear region of the galaxy, or the movement of the ionized gas in a starburst-driven outflow. The first possibility is discussed in Rodriguez-Rico et al. (2006), with the conclusion that it is unlikely (although not a priori rejectable) because of the lack of the characteristic S-shape in the velocity field for the secondary bar. Because the lack of an AGN does not imply the absence of a supermassive object, the second option cannot be ruled out by our conclusions that the maser is not related to an AGN.

The third option, an anomalous structure associated with outflow material seems viable. Starburst-driven outflows are indeed present in (starburst) galaxies like NGC 253, and they are produced by the mechanical energy released in the ISM from strong stellar winds and supernova explosions (e.g. Nakai et al. 1987, for M 82; Greve et al. 2000, for NGC 2146). In addition, since the main excitation mechanism for water masers has been singled out to be collisional pumping generated in shocks (e.g. Lo 2005), the maser in NGC 253 could be related to the outflow itself, sharing with its ionized gas component the blueshifted velocity. A further issue in favor of the outflow option is that the majority of kilomasers are indeed related to particularly intense star formation activity that is also the necessary ingredient for outflow phenomena. Hence, it is not surprising that the maser line velocity is more consistent with that of the ionized gas than with that of the other molecular tracers not participating in the outflows.

As a last point on the relation between the kinematics of different gas components and maser origin, it is worth mentioning that large cavities, like bubbles and superbubbles, are produced in galactic disks by powerful starburst events that are detectable both in the atomic (HI) and molecular (CO) gas distribution (e.g. Weiß et al. 1999, for the case of M 82). Very recently, Sakamoto et al. (2006) detected two shells or cavities interpreted as superbubbles (labeled SB1 and SB2) in the CO nuclear disk of NGC 253 , with diameters of $\sim 100$ pc each and velocity structures indicative of expansion velocities of $\sim 50 \mathrm{~km} \mathrm{~s}^{-1}$. The center of the superbubble SB1 is located South-West with respect to source TH4 and the masers. Although the projected distance is too large to relate it to the maser, the contribution of similar structures should be taken into account when considering the velocity field anomalies and, in any case, Sakamoto et al. (2006) emphasize the presence of disturbances and the strong kinematic impact of the nuclear starburst on the central molecular disk hosting the starburst. This can, by itself, qualitatively motivate the anomalous velocities in the masing gas. 


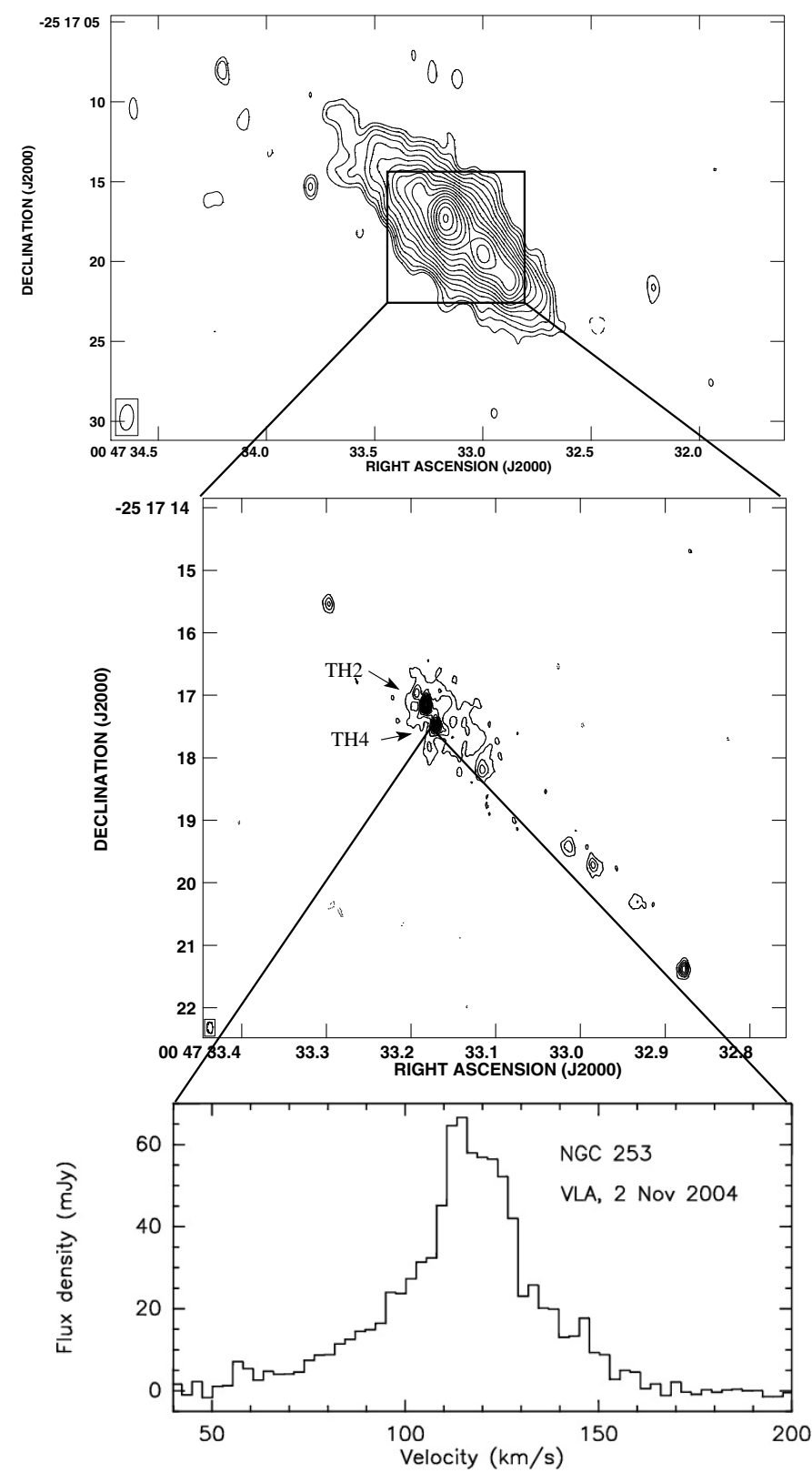

Fig. 2. Upper panel: C-array VLA image of NGC 253 at $22 \mathrm{GHz}$ observed on 2008 March 24. The contours start at $0.4 \mathrm{mJy}$ and increase by factors of $\sqrt{2}$. Middle panel: VLA A-array map of the continuum emission at $22 \mathrm{GHz}$ of NGC 253 observed on 2004 November 2. The synthesized beam is shown in lower left corner. Contours are at intervals of $0.45 \mathrm{mJy} /$ beam, beginning at $1.35 \mathrm{mJy} /$ beam $(3 \sigma)$. Lower panel: VLA A-Array spectrum of the water maser detected towards NGC 253 observed on 2004 November 2 . The channel spacing is $\sim 2.6 \mathrm{~km} \mathrm{~s}^{-1}$.

\section{Summary}

We have performed radio interferometic observations at $22 \mathrm{GHz}$ with the VLBA and the VLA of continuum sources and $\mathrm{H}_{2} \mathrm{O}$ masers in the nucleus of the galaxy NGC 253. The maser emission was found in two very compact (<330 AU) regions separated by $0.12 \mathrm{pc}$ and is almost surely related to star formation activity.
The continuum sources, while unresolved with the VLA, were not detected on mas scales with an upper limit of $1 \mathrm{mJy}$ $(5 \sigma)$. This questions the presence of a possible low-luminosity active galactic nucleus which has been previously assumed to explain the radio emission from the brightest radio source in the central part of NGC 253. Thus, the central region of NGC 253 is dominated by a starburst, and not by an AGN.

\section{References}

Anantharamaiah, K. R., \& Goss, W. M. 1996, ApJ, 466, L13

Barvainis, R., \& Antonucci, R. 2005, ApJ, 628, L89

Beasley, A. J., Gordon, D., Peck, A. B., et al. 2002, ApJS, 141, 13

Becker, R., Henkel, C., Wilson, T. L., \& Wouterloot, J. G. A. 1993, A\&A, 268, 483

Brunthaler, A., Falcke, H., Bower, G. C., et al. 2000, A\&A, 357, L45

Brunthaler, A., Falcke, H., Bower, G. C., et al. 2005a, A\&A, 435, 497

Brunthaler, A., Reid, M. J., Falcke, H., Greenhill, L. J., \& Henkel, C. 2005b, Science, 307, 1440

Brunthaler, A., Reid, M. J., \& Falcke, H. 2005c, Atmosphere-Corrected PhaseReferencing, in Future Directions in High Resolution Astronomy, ASP Conf. Ser., 340, 455

Brunthaler, A., Reid, M. J., Falcke, H., Henkel, C., \& Menten, K. M. 2007, A\&A, 462, 101

Canzian, B., Mundy, L. G., \& Scoville, N. Z. 1988, ApJ, 333, 157

Castangia, P., Tarchi, A., Henkel, C., \& Menten, K. M. 2008, A\&A, 479, 111

Churchwell, E., Witzel, A., Huchtmeier, W., et al. 1977, A\&A, 54, 969

Claussen, M. J., Diamond, P. J., Braatz, J. A., Wilson, A. S., \& Henkel, C. 1998, ApJ, 500, L129

Claussen, M. J., Goss, W. M., Frail, D. A., \& Seta, M. 1999, AJ, 117, 1387

Davidge, T. J., \& Pritchet, C. J. 1990, AJ, 100, 102

Fernández-Ontiveros, J. A., Prieto, M. A., \& Acosta-Pulido, J. A. 2008, MNRAS, 392, L16

Fey, A. L., Gaume, R. A., Nedoluha, G. E., \& Claussen, M. J. 1994, ApJ, 435, 738

Forbes, D. A., \& Depoy, D. L. 1992, A\&A, 259, 97

Gallimore, J. F., Henkel C., Baum S. A., et al. 2001, ApJ, 556, 694

Greenhill, L. J., Booth R. S., Ellingsen S. P., et al. 2003, ApJ, 590, 162

Greve, A., Neininger, N., Tarchi, A., \& Sievers, A. 2000, A\&A, 364, 409

Henkel, C., Tarchi, A., Menten, K. M., \& Peck, A. B. 2004, A\&A, 414, 117

Ho, P. T. P., Martin, R. N., Henkel, C., \& Turner, J. L. 1987, ApJ, 320, 663

Hofner, P., Baan, W. A., \& Takano, S. 2006, AJ, 131, 2074

Impellizzeri, C. M. V., McKean, J. P., Castangia, P., et al. 2008, Nature, 456, 927

Krichbaum, T. P., Zensus, J. A., Witzel, A., et al. 1993, A\&A, 274, L37

Lenc, E., \& Tingay, S. J. 2006, AJ, 132, 1333

Lo, K. Y. 2005, ARA\&A, 43, 625

Miyoshi, M., Moran, J., Herrnstein, J., et al. 1995, Nature, 373, 127

Mouhcine, M., Ferguson, H. C., Rich, R. M., Brown, T. M., \& Smith, T. E. 2005, ApJ, 633, 810

Nakai, N., Hayashi, M., Handa, T., et al. 1987, PASJ, 39, 685

O’Dea, C. P., \& Baum, S. A. 1997, AJ, 113, 148

Paglione, T. A. D., Tosaki, T., \& Jackson, J. M. 1995, ApJ, 454, L117

Peck, A. B., Henkel, C., Ulvestad, J. S., et al. 2003, ApJ, 590, 149

Peng, R., Zhou, S., Whiteoak, J. B., Lo, K. Y., Sutton, E. C. 1996, ApJ, 470, 821

Pina, R. K., Jones, B., Puetter, R. C., \& Stein, W. A. 1992, ApJ, 401, L75

Reid, M. J., \& Ho, P. T. P. 1985, ApJ, 288, L17

Reid, M. J., \& Brunthaler, A. 2004, ApJ, 616, 872

Reid, M. J., Braatz, J. A., Condon, J. J., et al. 2008, ApJ, in press [arXiv: 0811.4345]

Rekola, R., Richer, M. G., McCall, M. L., et al. 2005, MNRAS, 361, 330

Rodríguez-Rico, C. A., Goss, W. M., Zhao, J.-H., Gómez, Y., \& Anantharamaiah, K. R. 2006, ApJ, 644, 914

Sakamoto, K., Ho, P. T. P., Iono, D., et al. 2006, ApJ, 636, 685

Scalise, E., \& Braz, M. A. 1981, Nature, 290, 36

Scoville, N. Z., Soifer, B. T., Neugebauer, G., et al. 1985, ApJ, 289, 129

Slysh, V. I., Val'tts, I. E., Migenes, V., et al. 1999, ApJ, 526, 236

Tingay, S. J. 2004, AJ, 127, 10

Ulvestad, J. S., \& Antonucci, R. R. J. 1997, ApJ, 488, 621

Weaver, K. A., Heckman, T. M., Strickland, D. K., \& Dahlem, M. 2002, ApJ, 576, L19

Weiß, A., Walter, F., Neininger, N., \& Klein, U. 1999, A\&A, 345, L23

Yusef-Zadeh, F., Cotton, W., Wardle, M., Melia, F., \& Roberts, D. A. 1994, ApJ, 434, L63 